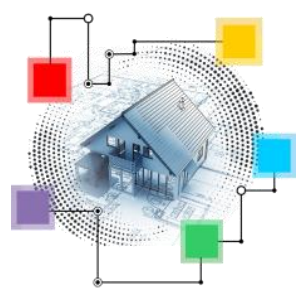

\author{
XIII SIMPÓSIO NACIONAL DE SISTEMAS PREDIAIS \\ DESEMPENHO E INOVAÇÃO \\ DE SISTEMAS PREDIAIS HIDRÁULICOS \\ SÃO PAULO - 04 DE OUTURO DE 2019
}

\title{
AVALIAÇÃO DO TAMANHO DAS SÉRIES HISTÓRICAS PARA APROVEITAMENTO DE ÁGUA PLUVIAL: UMA REVISÃO BIBLIOGRÁFICA
}

\section{EVALUATION OF THE LENGTH OF THE RAINFALL TIME SERIES FOR RAINWATER HARVESTING: A BIBLIOGRAPHIC REVIEW}

\author{
SILVA, Bruna Fioramonte'; CAMPOS, Marcus André Siqueira ${ }^{2 ;}$ \\ ${ }^{1}$ PPG-GECON/EECA/UFG, brunafioramonte@ hotmail.com \\ ${ }^{2}$ PPG-GECON/EECA/UFG, marcus_campos@ufg.br
}

\begin{abstract}
RESUMO
Os sistemas prediais de aproveitamento de água pluvial (SPAAPs) estão sendo cada vez mais utilizados como fonte alternativa a água potável. A série histórica de dados pluviométricos é um dos dados de entrada para o dimensionamento do reservatório de água pluvial, no entanto, muitas são as localidades que não possuem extensos dados de forma a garantir adequada estimativa da oferta. Assim, o objetivo deste estudo é expor como os estudos sobre a análise do tamanho da série histórica de dados pluviométricos foram conduzidos, por meio de revisão bibliográfica da literatura, identificando tendências de estudos futuros devido a lacunas do conhecimento. Foi realizada uma busca de artigos publicados na base de dados Scopus e, por meio de critérios de inclusão pré-definidos, foram selecionados 4 artigos aderentes. Foi observado que o tema começou a ser estudado recentemente e que seu estudo se concentra em apenas dois países. Com as análises realizadas, são fornecidas recomendações para trabalhos futuros e uma visão geral da literatura.
\end{abstract}

Palavras-chave: Séries pluviométricas, Aproveitamento de água pluvial, sistemas prediais.

\begin{abstract}
Rainwater harvesting $(R W H)$ systems are increasingly being used as an alternative source of potable water. The rainfall time series is one of the data inputs for the design of the reservoir, however, there are not many locations in order to guarantee an adequate estimate of the supply. Thus, the objective of this study is to show how the studies on the analysis of the size of the historical series of pluviometric data were conducted, through bibliographical review of the literature, identifying tendencies of future studies due to knowledge gaps. A search of articles published in the Scopus database was realized and, by pre-defined inclusion criteria, 4 relevant articles were selected. It was observed that the subject began to be studied recently and that its study concentrates in only two countries. With the analyzes carried out, recommendations are provided for future studies and an overview of the literature.
\end{abstract}

Keywords: Rainfall time series, Rainwater harvesting, Length of the rainfall time series, plumbing systems. 


\section{INTRODUÇÃO}

Os setores de fornecimento de suprimentos básicos, como por exemplo o de abastecimento de água potável, não conseguem acompanhar o aumento populacional acelerado, principalmente dos grandes centros urbanos. A escassez tem aumentado cada vez mais e os eventos de falta de água tem sido recorrentes; a exemplo disso, pode-se citar a crise ocorrida na cidade de São Paulo, Brasil, em 2015 e o "Dia Zero" na cidade do Cabo na África do Sul em 2018 (KELMAN; PINZAN, 2015).

O aumento da demanda por água e a oferta não correspondente tem motivado pesquisas voltadas a utilização de fontes que juntamente com a rede de abastecimento pública possa suprir a demanda e até mesmo diminuir o consumo de água potável para fins com qualidade diferente da necessária ou consumo humano (GERALDI; GHISI, 2010).

A utilização da água de chuva como alternativa à água potável é uma iniciativa que, juntamente com outras soluções que visem a conservação de água pode ser uma opção para a redução do consumo de água potável e atendimento da demanda (CAMPOS; ILHA, 2010).

Os dados pluviométricos, que são parte dos dados de entrada para o dimensionamento do reservatório de água pluvial, podem ser obtidos por meio de diversas bases de dados. No Brasil pode-se utilizar o Banco de Dados Meteorológicos para Ensino e Pesquisa (BDMET) e o Sistema de Monitoramento Agrometeorológico - Agritempo, contudo essas e outras bases disponíveis muitas vezes não possuem extensas séries históricas de dados pluviométricos que auxiliam na caracterização mais abrangente do perfil de chuva do local considerado e maior confiabilidade nas simulações para determinação do SPAAP (INMET, 2019).

A possibilidade de utilização de uma série histórica de dados pluviométricos curta, mas que possibilite adequada caracterização do perfil temporal da região, possibilitaria um dimensionamento mais sensível do reservatório de água pluvial. A importância da adequada caracterização da curva de precipitação é necessária pois está diretamente relacionada com a oferta.

Estudos relatados na literatura utilizaram diversos tamanhos de séries históricas para o dimensionamento do reservatório de água pluvial, muitos vezes dependente da disponibilidade dos dados. Analisar a influência do comprimento da série no processo de concepção do SPAAP se torna importante de forma a fundamentar o dimensionamento.

Diante disso, este trabalho tem como objetivo a realização de uma revisão bibliográfica para verificar a influência do tamanho das séries históricas de dados pluviométricos em sistemas de aproveitamento de água pluvial.

\section{MÉTODO}

Nas etapas iniciais de revisão do tema considerado foi observada falta de estudos específicos que determinassem o tamanho ótimo das séries históricas de dados pluviométricos para o dimensionamento do reservatório de água pluvial, dificultando uma revisão sistemática da literatura que fosse definitiva. 
Foi realizado um levantamento dos estudos a respeito da influência dos tamanhos das séries históricas de dados pluviométricos com o propósito de obter uma melhor compreensão de como essas análises foram conduzidas. Foram seguidas as etapas consideradas por Amos et al. (2018) e Pacheco e Campos (2016).

Inicialmente foram pensadas algumas questões que pudessem auxiliar durante a busca pelos artigos publicados. Assim, tentando responder alguns pontos a seguinte pergunta foi realizada:

- Quais pesquisas consideraram o tamanho da série histórica para o dimensionamento do reservatório do SPAAP?

As buscas foram realizadas, para este trabalho, apenas na base de dados Scopus, considerando apenas artigos de periódicos. A string de pesquisa adotada foi (rainfall AND time AND series) e (tank OR reservoir) podendo ser encontradas em títulos, resumos e palavras-chaves dos estudos existentes na base de dados.

Não se estabeleceu restrições quanto a área do conhecimento e ao ano de publicação. De forma a analisar se existiam artigos com o tema procurado em estudos de outras áreas e poder perceber a evolução temporal das pesquisas desenvolvidas.

De posse de todos os artigos encontrados, a primeira análise foi realizada pelos títulos dos artigos. Foram excluídos todos que não abordavam sobre reservatório de água pluvial e série histórica.

Com os artigos selecionados pela análise dos títulos, os resumos foram lidos e feita a segunda seleção, excluindo daqueles que não consideravam análise do tamanho da série histórica para o dimensionamento do SPAAP.

A terceira análise e exclusão foi por meio da leitura do texto completo. Os artigos que não estavam em língua inglesa foram descartados. Aqueles que não foi possível ter acesso ao texto completo, por estarem em periódicos não assinados pela Universidade, também foram excluídos.

Depois de selecionados todos os artigos que fariam parte do grupo aderente, foram extraídos os dados considerados relevantes para a análise: ano da publicação, palavraschave utilizadas, países onde ocorreram as pesquisas, tamanhos das séries históricas utilizadas e as considerações sobre a influência do tamanho da série.

Os dados coletados foram avaliados e resumidos, fornecendo um panorama atual do tema abordado.

\section{RESULTADOS E DISCUSSÕES}

Foram encontrados, com os critério pré-estabelecidos, 407 publicações na base de dados Scopus. Desse total, 4 publicações fizeram parte do grupo aderente como pode ser observado na Tabela 1. 
TABELA 1 - Critérios e resultados das seleções realizadas.

\begin{tabular}{ll}
\hline Artigos selecionados & +407 \\
Lendo os títulos & -398 \\
Lendo os resumos & -3 \\
Outras línguas & -1 \\
Acesso bloqueado & -1 \\
Artigos aderentes & +4 \\
\hline
\end{tabular}

Fonte: Os autores.

Foram quantificadas as palavras-chave de forma a visualizar quais foram as mais consideradas pelos autores dos artigos aderentes. A relação pode ser observada na Tabela 2.

TABELA 2 - Frequência de utilização de palavras-chave nos artigos aderentes.

\begin{tabular}{lc}
\hline Palavra-chave & Quantidade \\
\hline Potable water savings & 3 \\
Rainfall time series & 3 \\
Computer simulation & 2 \\
Rainwater tank sizing & 2 \\
Artigos aderentes & 1 \\
Rainwater & 1 \\
Rainwater harvesting & 1 \\
Rainwater harvesting system & 1 \\
Rainwater harvesting system (RWH) & 1 \\
Tank sizing & 1 \\
Time series & 1 \\
Urban water management & 1 \\
Water demand & 1 \\
\hline
\end{tabular}

Fonte: Os autores.

As publicações a respeito da influência do tamanho da série histórica de dados pluviométricos começou a ser realizada recentemente e teve um aumento de produção científica nos últimos 2 anos. Esse aumento de publicações provavelmente ocorreu devido à falta de séries históricas de dados pluviométricos extensas em diversas cidades. O Brasil foi o país com maior número de publicações seguido da Colômbia. Com relação ao Brasil, foi observado que as produções ocorreram por meio da mesma afiliação institucional (Tabela 3). 
TABELA 3 - Distribuição das publicações por ano e localidade.

\begin{tabular}{lll}
\hline Ano & País & Afiliação Institucional \\
\hline 2012 & Brasil & Universidade Federal de Santa Catarina \\
2017 & Brasil & Universidade Federal de Santa Catarina \\
2017 & Colômbia & Universidad de Caldas \\
2018 & Brasil & Universidade Federal de Santa Catarina \\
\hline
\end{tabular}

Fonte: Os autores.

Os resultados encontrados nestas pesquisas foram analisadas. Em Ghisi et al. (2012) foram analisados séries históricas de 1, 2, 3, 4, 5, 10, 20 e 30 anos de dados pluviométricos da cidade de Santa Bárbara do Oeste, estado de São Paulo, Brasil. As simulações foram realizadas por meio do software Netuno. Os autores verificaram que os tamanhos de 1 a 13 anos foram considerados adequados, contudo o tamanho da série histórica de dados pluviométricos depende da demanda de água de chuva. Em caso de baixa demanda, séries de um a dois anos fornecem resultados semelhantes aos de séries de longo prazo. Quanto maior a demanda e menor a série histórica, maior a dispersão encontrada das capacidades ideais dos reservatórios em relação às séries de longo prazo.

Já Geraldi e Ghisi (2017) avaliaram a possibilidade de utilização de séries históricas curtas de dados pluviométricos. Para isso, os autores utilizaram dados diários de precipitação da cidade de Berlim na Alemanha. Foram simulados os comprimentos de 1, $2,3,4,5,6,7,8,9,10,15,20$ e 30 anos e os resultados encontrados foram comparados com a série de referência de 30 anos. A ferramenta utilizada para as simulações foi novamente o software Netuno. Para que os tamanhos das séries históricas fossem considerados satisfatórios os resultados encontrados deveriam ser $95 \%$ equivalentes aos resultados da série de referência.

Os autores verificaram que a série histórica de dez anos foi a que mais se aproximou da série de referência de 30 anos, para uma maior capacidade de captação de água e potencial ideal de economia atendida.

Galarza-Molina e Torres (2017) propuseram um método simplificado para o dimensionamento de reservatório de água pluvial e utilizaram três tamanhos de séries históricas, 76 anos, últimos 10 e 5 anos. Houveram variações nos resultados encontrados e não puderam concluir a influência do tamanho da série histórica, tendo como hipótese das variações uma possível influência da alteração das precipitações ao longo do tempo. Os autores pretendem analisar, em estudos posteriores, as possíveis causas das variações dos resultados encontrados considerando possível mudança climática.

Geraldi e Ghisi (2018) avaliaram a possibilidade de utilização de séries históricas curtas. Eles utilizaram dados diários de treze cidades: Alexandria, no Egito; Barcelona, na Espanha; Berlim, na Alemanha; Dar-El-Beida, na Argélia; Darwin, na Austrália; Encarnación, no Paraguai; Moscou, na Rússia; Nova Iorque, nos Estados Unidos; Paris, na França; Quebec, no Canadá; Santarém e Santo Amaro, no Brasil; e Shanwei, na China. Os comprimentos e software utilizado foram os mesmos de Geraldi e Ghisi (2017). Os comprimentos foram considerados semelhantes, quando pelo menos $90 \%$ dos resultados 
encontrados para uma série história de curto prazo fossem equivalentes aos da séria de 30 anos.

Os autores concluíram em seu estudo que a série histórica de 9 anos foi a que forneceu mais resultados equivalentes aos obtidos com a série de referência de 30 anos para os casos estudados. Com os resultados encontrados, percebeu-se que várias séries de curto prazo podem garantir alguns resultados semelhantes aos da série de referência. Os autores concluíram também que a demanda de água pluvial não teve nenhuma influência considerável quando utilizados tamanhos diferentes de séries históricas.

\section{CONSIDERAÇÕES FINAIS}

Os resultados encontrados mostram a recente busca por parte da comunidade científica de se estudar a influência do tamanho da série histórica de dados pluviométricos no dimensionamento do SPAAP. Os resultados encontrados pelos autores se restringem aos locais estudados, devendo analisar o regime pluviométrico de cada região que se intencione projetar um SPAAP.

De acordo com os dados obtidos das análises, a possibilidade de utilização de séries históricas de dados pluviométricos mais curtas propiciaria, nos casos dos locais que possuem recente monitoramento pluviométrico, realizar o dimensionamento do SPAAP. Contudo, mostra-se necessária a avaliação do tamanho da série histórica de modo a garantir adequado dimensionamento.

Observa-se que há uma lacuna de conhecimento sobre este assunto, tornando necessário um número maior de pesquisas que visem analisar a influência do tamanho da série histórica, em especial no Brasil, com diferentes regimes pluviométricos para cada uma das regiões do país.

\section{REFERÊNCIAS}

AMOS, C. C.; RAHMAN, A.; KARIM, F.; GATHENYA, J. M. A scoping review of roof harvested rainwater usage in urban agriculture: Australia and Kenya in focus. Journal of Cleaner Production, v. 202, p. 174-190, 2018. DOI: 10.1016/j.jclepro.2018.08.108

CAMPOS, M. A. S.; ILHA, M. S. O. Dimensionamento de reservatórios para o aproveitamento de água pluvial: critérios econômicos. In: XIII ENCONTRO NACIONAL DE TECNOLOGIA DO AMBIENTE CONSTRUIIDO, 2010, Canela, RS. Anais... 2010.

GALARZA-MOLINA, S.; TORRES, A. Sizing method for stormwater harvesting tanks using daily resolution rainfall and water demand data sets. Revista Luna Azul, v. 45, p. 107 122, 2017. DOI: 10.17151/luaz.2017.45.7

GHISI, E.; CARDOSO, K. A.; RUPP, R. F. Short-term versus long-term rainfall time series in the assessment of potable water savings by using rainwater in houses. Journal of Environmental Management, v. 100, p. 109-119, 2012. DOI: 10.1016/j.jenvman.2011.12.031

GHISI, E.; GERALDI, M. S. Assessment of the length of rainfall time series for rainwater harvesting in buildings. Resources, Conservation and Recycling, v. 133, p. 231-241, 2018. DOI: 10.1016/j.resconrec.2018.02.007 
GHISI, E.; GERALDI, M. S. Influence of the length of rainfall time series on rainwater harvesting systems: A case study in Berlin. Resources, Conservation and Recycling, v. 125, p. 169-180, 2017. DOI: 10.1016/j.resconrec.2017.06.011

GHISI, E.; GERALDI, M. S. Parameters Influencing the sizing of rainwater tanks for use in houses. Water Resources Management, v. 24, p. 2381-2403, 2010. DOI: 10.1007/s112690099557-4

INMET (Instituto Nacional de Meteorologia). Dados históricos. Disponível em: < http://www.inmet.gov.br/portal/ > Acesso em: 01 de junho de 2019.

KELMAN; PINZAN,2015. Estratégias e Soluções da SABESP para a região metropolitana de São Paulo. São Paulo. Disponível em:

<http://site.sabesp.com.br/site/uploads/file/crisehidrica/chess_crise_hidrica.pdf> Acesso em: 01 de junho de 2019.

PACHECO, G. C. R; CAMPOS, M. A. S. Economic feasibility of rainwater harvesting systems: a systematic literature review. Journal of Water Supply: Research and Technology AQUA, v. 66, p. 1-14, 2016. DOI: 10.2166/aqua.2016.048 